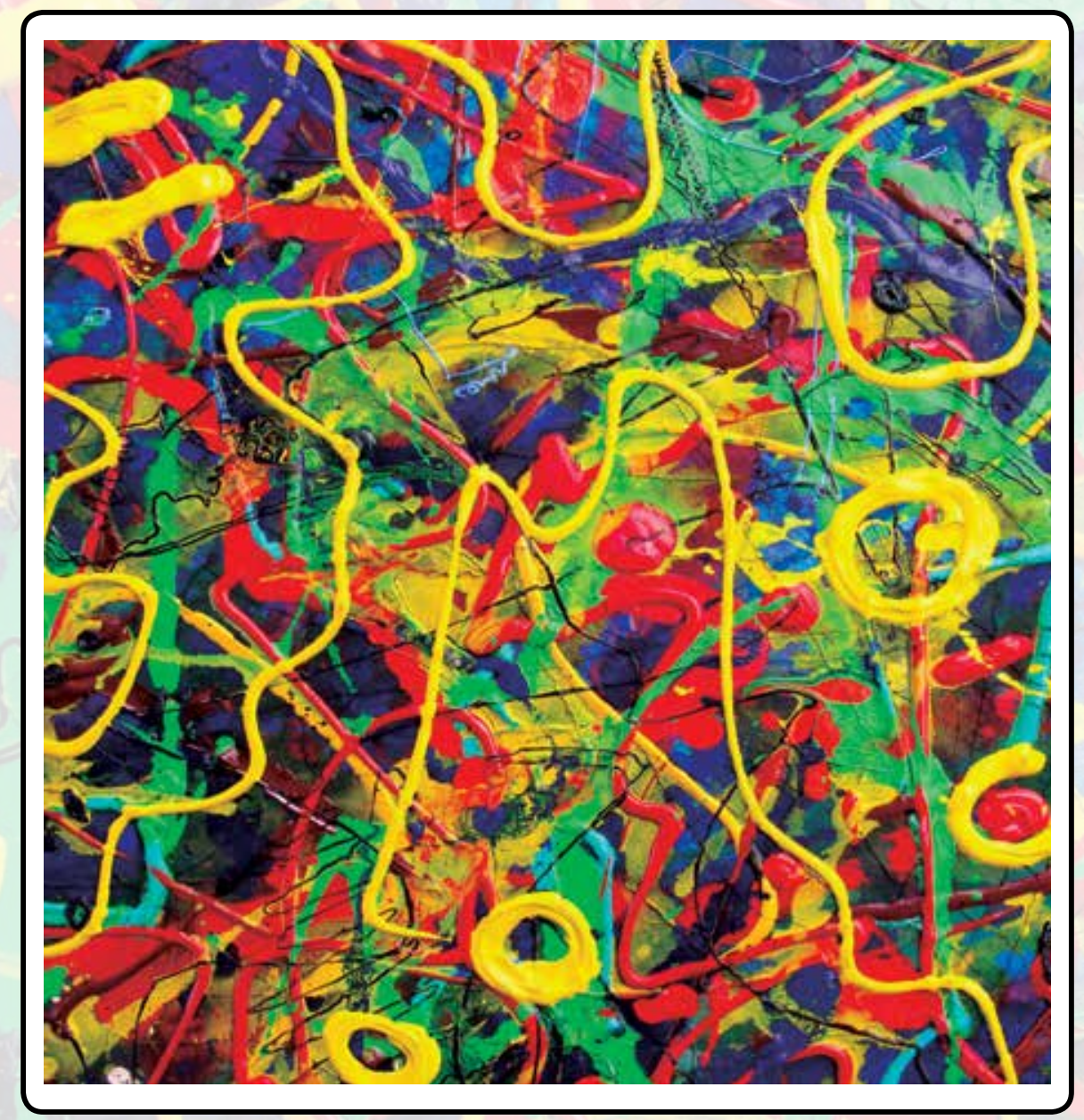

Camilo Umaña V. Sin título Acrílico sobre Lienzo 70 x 40 



\title{
ESTRATEGIAS DE ACCIÓN Y POTENCIAL COMPETITIVO DE EMPRESAS DE INTERMEDIACIÓN ADUANERA EN CARTAGENA DE INDIAS
}

\author{
ACTION STRATEGIES AND COMPETITIVE POTENTIAL OF CUSTOMS \\ BROKERAGE FIRMS IN CARTAGENA DE INDIAS
}

\author{
Juan David Sepúlveda Chaverral \\ Lourdes Meriño Stand ${ }^{2}$ \\ María Judith Vélez Murillo \\ Cristian Flórez Echenique
}

\begin{abstract}
Resumen
Este trabajo busca establecer las diferentes relaciones y tipologías existentes en torno al desarrollo de las estrategias competitivas existentes dentro del sector de las agencias de aduanas en la ciudad de Cartagena de Indias. Para ello, se ha desarrollado un estudio empírico mediante la aplicación de un modelo de evaluación de potencial competitivo a una muestra de 30 empresas del sector; con los resultados obtenidos fue posible realizar una valoración cualitativa, el análisis de concurrencia de las estrategias y los elementos que fundamentan el desarrollo del ideal competitivo dentro de este sector, se encontró con esto, que no existe un hilo conductor en el desarrollo de estrategias, con un alto grado de dispersión en el enfoque de gestión, y un modelo de seguimiento a competidores referenciados, así mismo, se logró demostrar que los factores de infraestructura, posicionamiento y satisfacción del cliente orientan el enfoque competitivo en dichas organizaciones.
\end{abstract}

\section{Palabras clave}

Potencial competitivo, Gestión, posicionamiento estratégico

Clasificación JEL: M19

\begin{abstract}
This paper aims to establish the different typologies and relationships around the competitive strategies in the customs broking companies in Cartagena de Indias. An empirical study was completed using a competitive potential evaluation model in a sample of thirty companies in this business area; the results allowed doing a qualitative evaluation of the strategies, the analysis of concurrence of the strategies and the elements that support the development of the competitive ideal within this sector. This showed that there is not a single approach in strategy development, and that there is a great dispersion in the management approach, and a follow-up model for the referenced competitors. It was also possible to find that infrastructure, positioning and customer satisfaction are the drivers of the competitive development of this companies.
\end{abstract}

\section{Keywords}

Competitive potential, Management, Strategic position

1 Maestría en Desarrollo Empresarial, Docente Investigador Universidad Manuela Beltrán (Colombia). Correo electrónico: juankarenium@gmail.com

2 Msc, Estudiante Doctorado en Ingeniería Química, Universidad Industrial de Santander (Colombia).

3 Ingeniera Industrial, Gerente Administrativa y Finanzas en Servicios Industriales Somonetti Colombia SAS Fundación Universitaria Tecnológico Comfenalco Cartagena (Colombia).

4 Ingeniero Industrial, Gerente Comercial- Representante Legal en CF Soluciones en madera SAS - Fundación Universitaria Tecnológico Comfenalco Cartagena (Colombia). 


\section{Introducción}

El presente trabajo presenta los resultados de la evaluación conjunta de un modelo de potencial competitivo aplicado a un grupo de empresas cuya actividad se centra en el proceso de intermediación aduanera en la ciudad de Cartagena de Indias, agrupadas en la Federación Colombiana de Transitarios, Intermediarios Aduaneros y Almacenadoras "FITAC". El propósito principal del mismo se enfoca en establecer las diferentes relaciones y tipologías existentes en torno al desarrollo de las estrategias competitivas planteadas dentro del sector estudiado.

Se aplicó a un total de 30 empresas un modelo denominado "metodología para la evaluación del potencial competitivo en pequeñas y medianas empresas basado en el esquema de capacidades internas", cuyos resultados muestran las conclusiones principales de análisis interno de las organizaciones a partir de los niveles de Gestión y Posicionamiento Estratégico Organizacional, con la evaluación conjunta de ocho variables que definen elementos de estrategia en el desarrollo competitivo de las organizaciones.

En la primera parte se presenta una aproximación teórica a los elementos que definen el modelo utilizado y el concepto de competitividad que fundamenta el desarrollo del trabajo, para luego presentar aspectos puntuales de la aplicación de las muestras en la descripción de la metodología utilizada y, con ello, abrir el paso a una tercera sección donde se presentan los resultados y las conclusiones principales del estudio.

\section{Referentes Teóricos}

Desde los años 80, el concepto de competitividad ha estado directamente relacionado con el desarrollo de la capacidad de mercado de los productos. Diversos autores, además de apoyar estas ideas, han planteado que la competititividad se relaciona directamente con la capacidad de las empresas de enfrentar a sus competidores y vencerlos (Nurbel, 2007; Michalet, 1981).

Para Porter (2008) lo más importante en el desarrollo competitivo de las organizaciones, es enfocarse en garantizar la supervivencia de las empresas en el largo plazo. A su vez Krugman (1994), a partir de las distintas definiciones existentes sobre competitividad, plantea como necesario que los trabajos desarrollados en torno al concepto, sean explícitos en definir los elementos que abordan en el análisis.

En términos de su medición, en los últimos años la producción de literatura sobre el tema se ha multiplicado y los autores han planteado diversos enfoques metodológicos. 
Para el desarrollo de este trabajo, se ha tomado en cuenta el modelo propuesto por Sepúlveda y otros (2010) quienes plantean - desde el concepto y las posibilidades de medición - que la competitividad, ya vislumbrada desde la revolución industrial, se ha convertido en una necesidad empresarial cuya importancia e impacto, a medida que ha pasado el tiempo, exige mayor esfuerzo, educación, planeación a corto y largo plazo y la definición de aspectos o actividades que realmente agreguen valor a los productos y servicios al menor costo y con la mejor calidad. Lo anterior ha tomado mayor impulso a raíz de la aparición del fenómeno de la globalización el cual ha obligado a todas las empresas, en especial a los sectores mundiales de manufactura y de servicio, a trabajar y buscar el mejoramiento continuo de sus operaciones o, de lo contrario, enfrentar la extinción de la empresa como tal.

Si se tiene en cuenta que un país es competitivo cuando sus empresas son competitivas, la importancia de la competitividad radica en que se deben ofrecer productos y servicios que atiendan de manera confiable, accesible y segura y con la programación adecuada, las necesidades del cliente lo cual sólo se logra cuando las empresas se comprometen a ser competitivas en el contexto mundial.

Actualmente, los modelos de medición institucional de la competitividad se basan en el esquema de ranking comparativo, ante lo cual, para una empresa es necesario disponer de un marco de información complejo de sus competidores a un nivel de detalle que hace difícil su implementación en el escenario de las pequeñas y medianas empresas (PYMES). De esta manera se evidencia la necesidad de un cambio de enfoque que permita a estas unidades empresariales contar con un marco de análisis de sus capacidades competitivas, en relación a sus condiciones particulares.

Con relación a este punto, en el 2010 se definió una propuesta de modelo que intentaba superar los aspectos mencionados anteriormente al permitir, al mismo tiempo, una evaluación y autoevaluación de los factores principales de potencial competitivo enfocado en PYMES (Alomoto, 2010; Ríos, 2011; Sepúlveda et al, 2010). El modelo propuesto consta de dos factores (Gestión y Posicionamiento estratégico), ocho componentes y 36 variables. El modelo completo se muestra en la Tabla 1, a la Tabla original propuesta por Sepúlveda, Meriño, Del Río y García (2010) se ha agregado una columna donde se incluyen fuentes adicionales de soporte a cada componente analizado, para buscar establecer su utilidad en la aplicación del modelo en organizaciones de mayor tamaño. Así mismo, en la evaluación de cada componente, en el capítulo de resultados, estas referencias se utilizan como soporte comparativo y de referencia para el análisis. 
Tabla 1. Componentes, variables y condiciones en el modelo de medición de potencial competitivo

\begin{tabular}{|c|c|c|c|}
\hline Componente & Variable & Condición & \\
\hline \multirow{4}{*}{ Vigilancia } & Búsqueda & $\begin{array}{l}\text { ¿La empresa realiza PERIÓDICAMENTE un proceso } \\
\text { de búsqueda de información y vigilancia de sus } \\
\text { competidores, su entorno y los elementos que podrían } \\
\text { afectarla? }\end{array}$ & \multirow{4}{*}{$\begin{array}{l}\text { Sepúlveda Chaverra, } \\
\text { Meriño Stand, \& } \\
\text { Angulo Cuentas, 2010 }\end{array}$} \\
\hline & Análisis & \begin{tabular}{|l|} 
¿Existe personal responsable del análisis de la \\
información derivada del proceso de vigilancia?
\end{tabular} & \\
\hline & Fuentes & $\begin{array}{l}\text { ¿La empresa utiliza para el análisis de la información } \\
\text { la totalidad de las siguientes fuentes: Internet, } \\
\text { Patentes, Estudios sectoriales, Boletines técnicos, } \\
\text { Boletines científicos? }\end{array}$ & \\
\hline & Conocimiento & $\begin{array}{l}\text { ¿Se generan y discuten en Comité informes de } \\
\text { resultados del proceso de vigilancia y ellos conducen } \\
\text { a la toma de decisiones sobre el negocio? }\end{array}$ & \\
\hline \multirow{4}{*}{$\begin{array}{l}\text { Toma de } \\
\text { Decisiones }\end{array}$} & $\begin{array}{l}\text { Bases } \\
\text { (Técnicas) }\end{array}$ & $\begin{array}{l}\text { ¿La toma de decisiones corresponde a un proceso } \\
\text { técnico de análisis de datos e información relevante } \\
\text { y no a un proceso basado en mitos y creencias de las } \\
\text { directivas? }\end{array}$ & \multirow{4}{*}{$\begin{array}{l}\text { Esser, Hillebrand, } \\
\text { Messner, \& Meyer- } \\
\text { Stamer, 1996; García, } \\
\text { Bolivar, \& Roa, } 2011\end{array}$} \\
\hline & Claves & \begin{tabular}{|l|} 
Se conocen y se evalúan PERIÓDICAMENTE los \\
factores claves de desempeño del negocio \\
\end{tabular} & \\
\hline & Experiencia & $\begin{array}{l}\text { El personal involucrado en la toma de decisiones } \\
\text { ha estado involucrado en el negocio y ha recibido } \\
\text { formación relacionada con el mismo durante los } \\
\text { últimos cinco (5) años }\end{array}$ & \\
\hline & Propiedad & $\begin{array}{l}\text { Cada persona en la organización conoce y aplica } \\
\text { sus alcances y limitaciones en el proceso de toma de } \\
\text { decisiones }\end{array}$ & \\
\hline \multirow{5}{*}{ Infraestructura } & Política & $\begin{array}{l}\text { Existe en la organización una política clara relacionada } \\
\text { con la infraestructura y el mantenimiento de la planta } \\
\text { física y equipos y además está de acuerdo a las normas } \\
\text { legales que rigen el sector. }\end{array}$ & \multirow{5}{*}{$\begin{array}{l}\text { Sarmiento Paredes, } \\
\text { Sánchez Sánchez, \& } \\
\text { Cruz García, 2009 }\end{array}$} \\
\hline & Beneficios & $\begin{array}{l}\text { Se conocen los beneficios de la infraestructura } \\
\text { actual en cuanto a los siguientes aspectos: cercanía } \\
\text { a los mercados, facilidades de acceso, cercanía y } \\
\text { facilidades con los proveedores, costos y ventajas } \\
\text { intangibles }\end{array}$ & \\
\hline & Condicionamiento & $\begin{array}{l}\text { Las condiciones de operación de la planta física y los } \\
\text { equipos de trabajo están de acuerdo a los parámetros } \\
\text { legales vigentes }\end{array}$ & \\
\hline & Aprovechamiento & $\begin{array}{l}\text { Se explotan económica y comercialmente los } \\
\text { beneficios de la infraestructura actual en cuanto a } \\
\text { los siguientes aspectos: cercanía a los mercados, } \\
\text { facilidades de acceso, cercanía y facilidades con los } \\
\text { proveedores, costos y ventajas intangibles }\end{array}$ & \\
\hline & Mejoras & $\begin{array}{l}\text { Existe un plan PERIÓDICO de mantenimiento, } \\
\text { adecuación, expansión y mejora de la planta física y } \\
\text { equipos de trabajo y además se cuenta con el capital } \\
\text { para su ejecución }\end{array}$ & \\
\hline
\end{tabular}




\begin{tabular}{|c|c|c|c|}
\hline Componente & Variable & Condición & \\
\hline \multirow{5}{*}{$\mathrm{I}+\mathrm{D}$} & Capital & $\begin{array}{l}\text { Existe en la organización una asignación económica } \\
\text { para los procesos de investigación e innovación }\end{array}$ & \multirow{5}{*}{$\begin{array}{l}\text { Raymond \& St-Pierre, } \\
2010\end{array}$} \\
\hline & Objetivos & $\begin{array}{l}\text { Existe una política y se evalúa PERIÓDICAMENTE } \\
\text { respecto a los cambios en productos y procesos y } \\
\text { estos no obedecen a simples requerimientos del } \\
\text { mercado y oportunidades esporádicas de acción }\end{array}$ & \\
\hline & $\begin{array}{l}\text { Encadena } \\
\text { miento }\end{array}$ & $\begin{array}{l}\text { En los procesos de investigación y desarrollo de } \\
\text { nuevos productos y servicios se CONOCEN Y } \\
\text { ESTABLECEN acuerdos de cooperación con los } \\
\text { siguientes actores: universidades y centros de } \\
\text { desarrollo tecnológico, competidores, proveedores y } \\
\text { clientes, consultores especializados }\end{array}$ & \\
\hline & Capacidades & $\begin{array}{l}\text { Existe en la organización una base de datos que } \\
\text { involucra las competencias individuales del personal } \\
\text { y sus capacidades en el proceso de investigación } \\
\text { y desarrollo de nuevos o mejorados productos y } \\
\text { procesos }\end{array}$ & \\
\hline & TIC & $\begin{array}{l}\text { La empresa cuenta con los siguientes recursos } \\
\text { relacionados con las TIC y los explota: } \\
\text { Internet banda ancha y planes de telefonía celular } \\
\text { nacional e internacional, bases de datos e información } \\
\text { especializada, programas de seguridad informática, } \\
\text { paquetes básicos de procesamiento de datos e } \\
\text { información y programas especializados para diseño, } \\
\text { y gestión de acuerdo a las tendencias del sector }\end{array}$ & \\
\hline \multirow{4}{*}{$\begin{array}{l}\text { Evaluación } \\
\text { financiera }\end{array}$} & Métodos & $\begin{array}{l}\text { Existe una metodología de evaluación y toma de } \\
\text { decisiones basada en el análisis de los estados } \\
\text { financieros y cumple con las siguientes condiciones: } \\
\text { Es aplicada periódicamente, involucra personal } \\
\text { de diferentes niveles en su análisis, la evaluación } \\
\text { involucra conceptos de análisis financiero actuales }\end{array}$ & \multirow{4}{*}{ Porter, 2008} \\
\hline & Enfoque & $\begin{array}{l}\text { El análisis financiero es una herramienta de apoyo } \\
\text { a la toma de decisiones que involucra un proceso } \\
\text { constante de capacitación en dichos aspectos de los } \\
\text { involucrados de manera que el proceso se encuentra } \\
\text { al día con las tendencias de evaluación financieras } \\
\text { globales }\end{array}$ & \\
\hline & Seguimiento & $\begin{array}{l}\text { Se realiza un seguimiento constante a los estados } \\
\text { financieros y los resultados de los ejercicios contables } \\
\text { mediante indicadores y tecnología y el personal tiene } \\
\text { acceso a los mismos de manera que pueda participar } \\
\text { en el proceso }\end{array}$ & \\
\hline & Finanzas & $\begin{array}{l}\text { La empresa ha tenido resultados financieros positivos } \\
\text { y cuenta con recursos para inversión y mejoramiento } \\
\text { continuo }\end{array}$ & \\
\hline
\end{tabular}




\begin{tabular}{|c|c|c|c|}
\hline Componente & Variable & Condición & \\
\hline \multirow{5}{*}{ Posicionamiento } & Liderazgo & $\begin{array}{l}\text { La empresa es reconocida por los clientes potenciales } \\
\text { y su imagen es positiva }\end{array}$ & \multirow[t]{5}{*}{ Porter, 2008} \\
\hline & Logística & $\begin{array}{l}\text { Existe un proceso de encadenamiento logístico con } \\
\text { clientes y proveedores que permite obtener ventajas } \\
\text { competitivas frente a otros competidores }\end{array}$ & \\
\hline & Valor & $\begin{array}{l}\text { Existe una metodología para la evaluación de valor } \\
\text { y se conocen las actividades generadoras del mismo } \\
\text { dentro de la organización y además son aplicadas }\end{array}$ & \\
\hline & Personal & $\begin{array}{l}\text { El personal de la organización cuenta con las } \\
\text { siguientes características: } \\
\text { Posee la formación necesaria para sus labores. } \\
\text { Tiene acceso a cursos y programas de formación } \\
\text { y actualización brindados por la organización. } \\
\text { Posee un contrato de acuerdo a sus capacidades y la } \\
\text { remuneración se encuentra al menos en el promedio } \\
\text { del sector. Conoce sus tareas y responsabilidades } \\
\text { y están consignadas en una manual de funciones o } \\
\text { perfil de competencias. Es tratado de acuerdo a su } \\
\text { condición de ser humano y en igualdad de condiciones } \\
\text { frente a sus superiores y subalternos }\end{array}$ & \\
\hline & Globalización & $\begin{array}{l}\text { La empresa conoce sus limitaciones y fortalezas } \\
\text { frente a la competencia global y ha establecido } \\
\text { planes periódicos de fortalecimiento organizacional, } \\
\text { técnico, tecnológico y frente a los mismos }\end{array}$ & \\
\hline \multirow{3}{*}{$\begin{array}{l}\text { Satisfacción del } \\
\text { cliente }\end{array}$} & Mercadeo & $\begin{array}{l}\text { Se conocen y utilizan los diferentes canales de } \\
\text { acceso a los clientes y la información respecto a ellos } \\
\text { permanece actualizada, además: } \\
\text { Es posible competir en precio. Es posible captar } \\
\text { nuevos clientes de la competencia. Existe un proceso } \\
\text { de fidelización de clientes }\end{array}$ & \multirow[t]{3}{*}{$\begin{array}{l}\text { Sarmiento Paredes, S., } \\
\text { Sánchez Sánchez, A., } \\
\text { \& Cruz García, M. G, } \\
2009\end{array}$} \\
\hline & Servicios & $\begin{array}{l}\text { Existe un proceso de valor agregado a los productos } \\
\text { y servicios ofertados mediante una estrategia de } \\
\text { servicio que incluya: } \\
\text { Sistema de comunicación de quejas y reclamos. } \\
\text { Seguimiento permanente a las indicaciones y } \\
\text { requerimientos de los clientes. Garantía y servicio } \\
\text { posventa }\end{array}$ & \\
\hline & Calidad & $\begin{array}{l}\text { Existe un proceso para la medición y aseguramiento } \\
\text { de la calidad de los productos y servicios ofertados } \\
\text { que involucra: } \\
\text { Sistema de control estadístico de la calidad. } \\
\text { Sistema de gestión de la calidad (En operación, no } \\
\text { necesariamente asegurado). Seguimiento de quejas, } \\
\text { reclamos y requerimientos de los clientes. Plan de } \\
\text { mejora de la calidad. Conocimiento y aplicación de } \\
\text { los estándares de calidad establecidos legalmente }\end{array}$ & \\
\hline
\end{tabular}




\begin{tabular}{|c|c|c|c|}
\hline Componente & Variable & Condición & \\
\hline \multirow{6}{*}{$\begin{array}{l}\text { Planeación } \\
\text { estratégica }\end{array}$} & Recursos & $\begin{array}{l}\text { La empresa conoce y explota los diferentes recursos } \\
\text { y Talento humano disponibles para el logro de sus } \\
\text { objetivos estratégicos }\end{array}$ & \multirow{6}{*}{$\begin{array}{l}\text { García, Bolivar, \& } \\
\text { Roa, } 2011\end{array}$} \\
\hline & Sistema & $\begin{array}{l}\text { Existe un sistema de planeación que involucra al } \\
\text { personal en todos los niveles de la organización }\end{array}$ & \\
\hline & Organización & $\begin{array}{l}\text { El modelo organizacional está acorde a las } \\
\text { tendencias del sector a nivel regional, nacional e } \\
\text { internacional }\end{array}$ & \\
\hline & Misión & $\begin{array}{l}\text { La misión de la organización permite la expansión } \\
\text { del negocio dentro del sector y al mismo tiempo } \\
\text { marca un derrotero a seguir que involucra los } \\
\text { siguientes aspectos: } \\
\text { Tecnología. Talento humano. Conocimiento del } \\
\text { sector y la industria. Tendencias globales. Sinceridad } \\
\text { en los postulados }\end{array}$ & \\
\hline & Visión & $\begin{array}{l}\text { La visión de la organización está presente en los } \\
\text { objetivos estratégicos }\end{array}$ & \\
\hline & Cambio & $\begin{array}{l}\text { La estructura organizacional, el modelo directivo, } \\
\text { y la actitud de los empleados permiten una eventual } \\
\text { disposición positiva frente a cambios en el entorno }\end{array}$ & \\
\hline
\end{tabular}

Fuente: adaptación de Sepúlveda, Meriño, Del Río \& García (2010)

\section{Metodología}

Este trabajo corresponde a la aplicación de un estudio de tipo descriptivo que tiene como propósito principal establecer las diferentes relaciones y tipologías existentes en torno al desarrollo de las estrategias competitivas en un conjunto de empresas del sector aduanero de la ciudad de Cartagena de Indias, dedicadas a actividades de intermediación aduanera, el objetivo del análisis persigue realizar una valoración individualizada de las estrategias y de la manera como éstas afectan el conjunto y concurren de acuerdo a algún parámetro especial dentro del comportamiento del sector. El modelo de evaluación denominado "metodología para la evaluación del potencial competitivo en pequeñas y medianas empresas basado en el esquema de capacidades internas" fue aplicado a un total de 30 empresas cuya actividad se centra en el proceso de intermediación aduanera en la ciudad de Cartagena de Indias agrupadas en la Federación Colombiana de Transitarios, Intermediarios Aduaneros y Almacenadoras "FITAC" y clasificadas en los niveles 1 y 2 de acuerdo al decreto 2883 de 2008.

La elección de la muestra analizada parte de un total de 61 empresas registradas en FITAC al año 2011, mediante parámetros estadísticos se definió una muestra inicial de 53 empresas, con un Nivel de confianza del 95\% y un intervalo de 5\%. Sin embargo, al momento de realizar el proceso de muestreo, se decidió realizar la evaluación por conveniencia utilizando como referentes a las 30 principales agencias, a partir de criterios de edad (aproximadamente el 32\% organizaciones tenían entre 
cero (0) y dos (2) años de constituidas), dinámica de participación en la organización e influencia en el sector, factores que podrían generar ruido en el proceso de muestreo y los resultados, tenida en cuenta la dinámica individual que representan.

El modelo de análisis seleccionado obedece a una construcción cualitativa sin competencia intra-sectorial, de ranking relativo y con características comparativas. Esto quiere decir que, al mismo tiempo, puede ser aplicado para el desarrollo de análisis interno de las organizaciones (Ríos Montañez, 2011) y para el análisis de grupos de empresas de manera comparada (Alomoto Bansui, 2010). Para la ejecución del estudio, el modelo propuesto involucra, como ya se dijo, dos dimensiones de análisis (Gestión y Posicionamiento estratégico) medidos a partir de la aplicación de diversos factores ideales de estrategia competitiva, evaluados en los niveles estratégicos organizacionales mediante un cuestionario que permite obtener una dimensión de análisis cualitativo y otra de tipo cuantitativo.

De esta forma, en la dimensión cualitativa se realizó un análisis gráfico de los resultados, a partir de los obtenidos en cada empresa de la muestra analizada. Cada componente (Factores del modelo) fue graficado en un diagrama radial mediante una plantilla de evaluación montada en el programa Microsoft Excel. El proceso fue diseñado de manera que permitiera establecer en una figura el comportamiento, por una parte de cada componente del modelo a nivel de sus variables y, por otra, la evaluación conjunta del modelo, a partir de sus componentes. Para contrastar los resultados obtenidos, se aplicó un análisis de correspondencias a los resultados del modelo completo, con el fin de establecer la forma de correspondencia entre las variables analizadas con el modelo en su conjunto, y con ello identificar la existencia de conjuntos estratégicos dentro de la muestra estudiada.

\section{Resultados}

A continuación se presentan los resultados de la aplicación del análisis de potencial competitivo en el sector de agencias de aduanas en Cartagena de Indias, que se muestran en dos niveles: los resultados que conducen al análisis cualitativo de las estrategias, considerado el factor Gestión, que incluye las variables de toma de decisiones, I+D, vigilancia, e infraestructura; y, el factor Posicionamiento estratégico, que incluye la evaluación financiera, la satisfacción del cliente y la planeación estratégica.

\subsection{Análisis Cualitativo de Resultados de Estrategia}

\subsubsection{Factor de Gestión}

\subsubsection{Toma de decisiones}


La toma de decisiones, como una estrategia de innovación social en las organizaciones, es la base para la estructuración de nuevos modelos de negocio y desarrollo organizacional (Esser, Hillebrand, Messner \& Meyer-Stamer, 1996) al tiempo que, si se basa en un conocimiento pleno del entorno de la organización, se constituye en una fuente de ventaja sostenible (García, Bolívar \& Roa, 2011)

Figura 2. Toma de decisiones

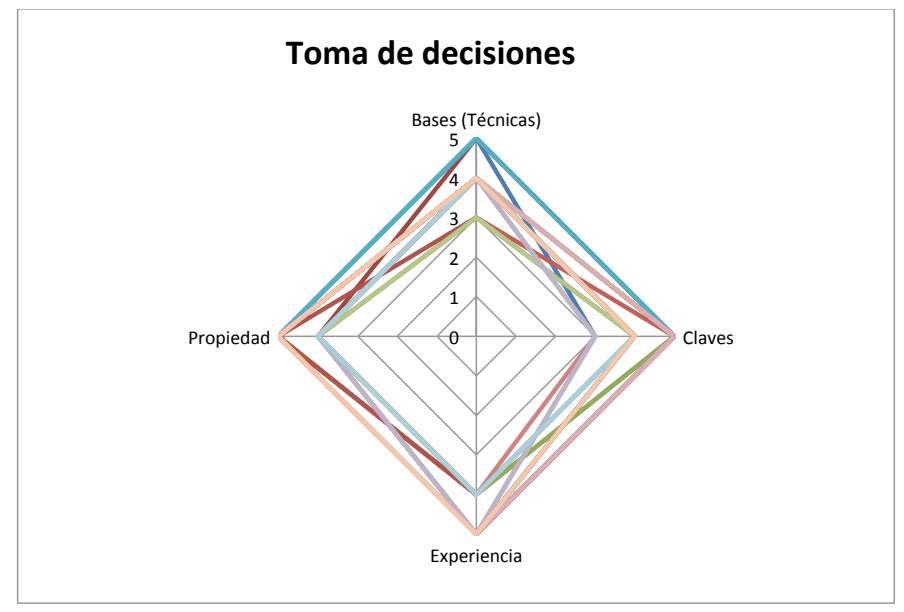

Fuente: Elaboración propia con base en los resultados del estudio

La toma de decisiones dentro de la estrategia competitiva, involucra el proceso mediante el cual se convierten datos e información en conocimiento (Bases). Igualmente, la periodicidad del proceso de análisis, sumado al conocimiento y la importancia para la organización de sus factores de éxito (Claves), así como el nivel de preparación, la experiencia y el conocimiento del negocio del personal involucrado en el análisis de la información (Experiencia) y el nivel de empoderamiento, apropiación y difusión de la información para la toma de decisiones (Propiedad) (Sepúlveda Chaverra, Meriño Stand, Del Río Cortina \& García, 2010).

En el caso analizado, para el conjunto de empresas estudiadas, cabe destacar que la toma de decisiones corresponde a un componente de alto nivel de importancia, con una mínima evaluación de 3, en las variables de Claves y Bases, lo que evidencia que dentro del desarrollo estratégico de las agencias de aduanas se presta mayor atención a la experiencia del personal para enfrentar los diferentes eventos de su actividad, tal como se puede apreciar en la Figura 2. El análisis al grupo de empresas estudiado permitió evidenciar también que, de acuerdo a la estrategia, las empresas se dividen, al menos, en dos grupos: por una parte las empresas con unidad de mando por sus condiciones locales y su origen, y por otra, aquellas que sólo son sedes de una organización matriz ubicada en otra ciudad, cuyo direccionamiento estratégico 
se encuentra supeditado a la dirección general y sus labores tienen un mayor grado de injerencia operativa.

\subsubsection{I I D}

Dentro del proceso innovador en las organizaciones, las actividades de I+D constituyen un esfuerzo positivo en el desarrollo de productos de valor agregado, cuyos resultados son transmitidos a los clientes (Raymond \& St-Pierre, 2010).

\section{Figura 3. I+D}

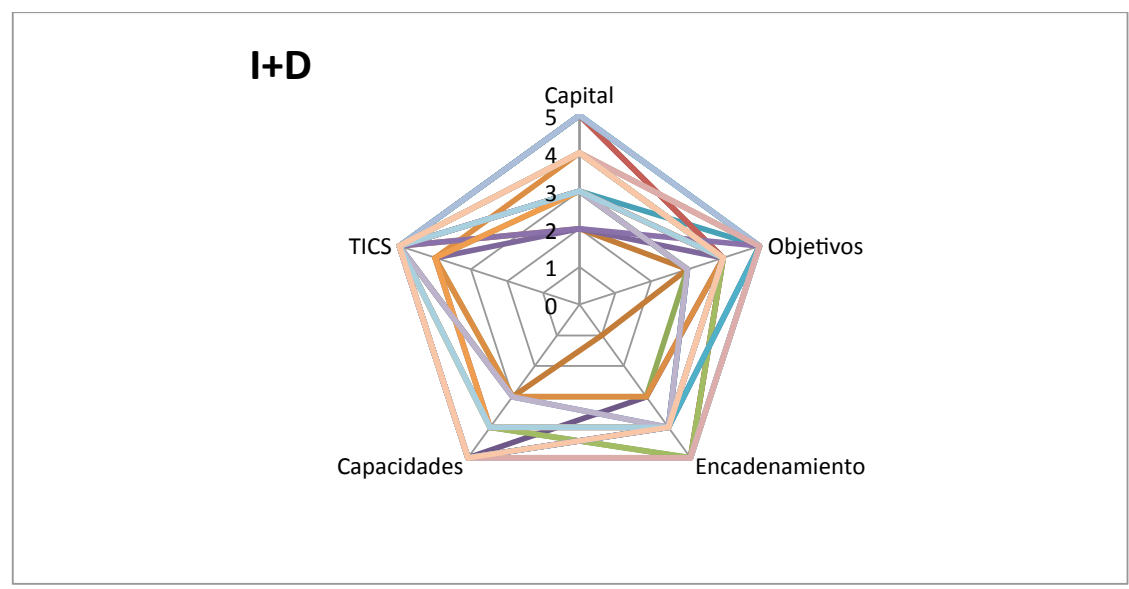

Fuente: Elaboración propia con base en los resultados del estudio

De acuerdo al modelo utilizado las actividades de $\mathrm{I}+\mathrm{D}$, dentro de la estrategia competitiva de las organizaciones incluyen: la existencia de una política de I+D organizacional que oriente los procesos innovadores (Objetivos), al tiempo que se desarrollan acciones integradas y apalancadas en la participación de otros agentes del entorno (Encadenamiento), con la destinación de recursos necesarios para la ejecución de las acciones planificadas (Capital) en un marco de conocimiento y apropiación de las competencias de su talento humano (Capacidades) y con la utilización de recursos tecnológicos apropiados a las acciones requeridas (TIC).

Para el caso de la I+D - cuyos resultados se pueden apreciar en la Figura 3- en el sector de agencias de aduanas en la ciudad de Cartagena de Indias, se observa un alto grado de variabilidad. Mientras se evidencia una tendencia en el desarrollo de acciones basadas en la utilización de las TIC, las demás dimensiones de la I+D no permiten visualizar una única estrategia en torno a esta variable. 


\subsubsection{Infraestructura}

De acuerdo con Sarmiento et al. (Sarmiento Paredes, Sánchez Sánchez \& Cruz García, 2009), dentro del proceso de evaluación de factores competitivos en el modelo de Porter, la Infraestructura, como parte del análisis de condiciones de los factores, determina una de las aristas del diamante competitivo que involucra los elementos físicos e intangibles que dan valor a la producción.

Figura 4. Infraestructura

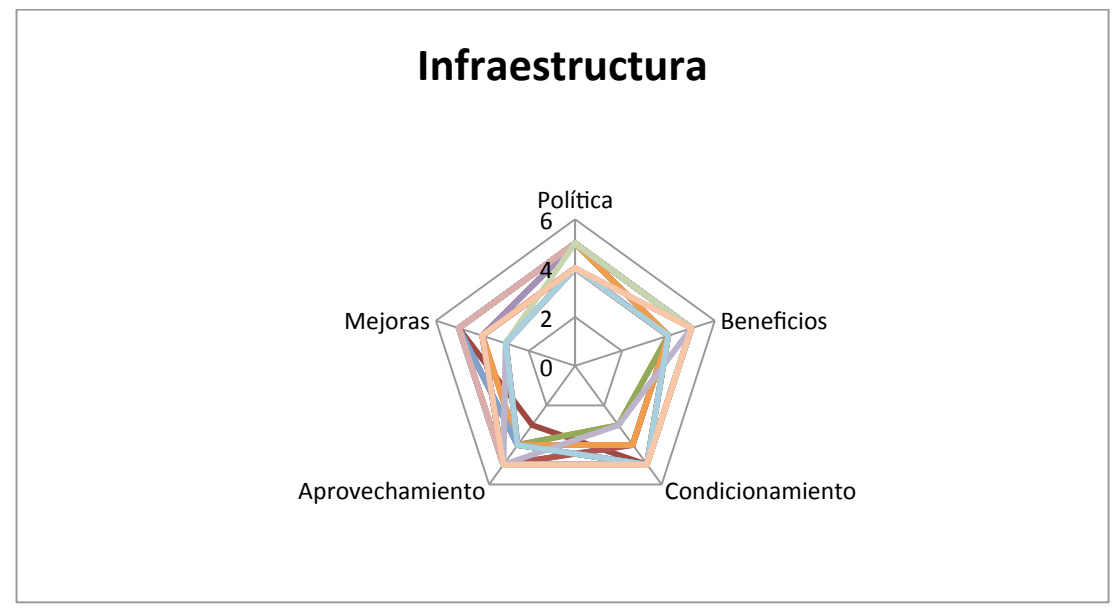

Fuente: Elaboración propia con base en los resultados del estudio

Dentro del sector de agencias de aduanas, cabe destacar que los resultados evidencian que la infraestructura representa a nivel global un elemento dominante dentro de la estrategia de desarrollo de potencial competitivo en las organizaciones. Esta variable muestra un significativo acercamiento hacia las puntas del pentágono, y se evidencia que hay un interés propio de las organizaciones en obtener la máxima calificación (Figura 4). De esta manera, el análisis de los resultados, complementado con procesos de entrevistas en FITAC, contribuye a mostrar la dinámica que tienen las empresas hacia este importante pilar del sector. No toda la muestra apunta a destacar el factor infraestructura como el más importante, pero si deja ver que todas intentan mejorar su posicionamiento en el mercado a través de este factor. Para este tipo de empresas, se convierte en obligatorio el contar con una infraestructura adecuada para la resolución de problemas y para la excelente prestación del servicio. 


\subsubsection{Vigilancia Tecnológica}

Las actividades de vigilancia tecnológica permiten no sólo convertir el conocimiento que existe en el entorno de la organización en conocimiento útil a la misma, sino que, al mismo tiempo, favorecen que esta pueda reaccionar anticipadamente a los cambios de tipo social, político y tecnológico que puedan afectar el desarrollo de sus actividades (Sepúlveda Chaverra, Meriño Stand, \& Angulo Cuentas, 2010).

Figura 5. Vigilancia Tecnológica

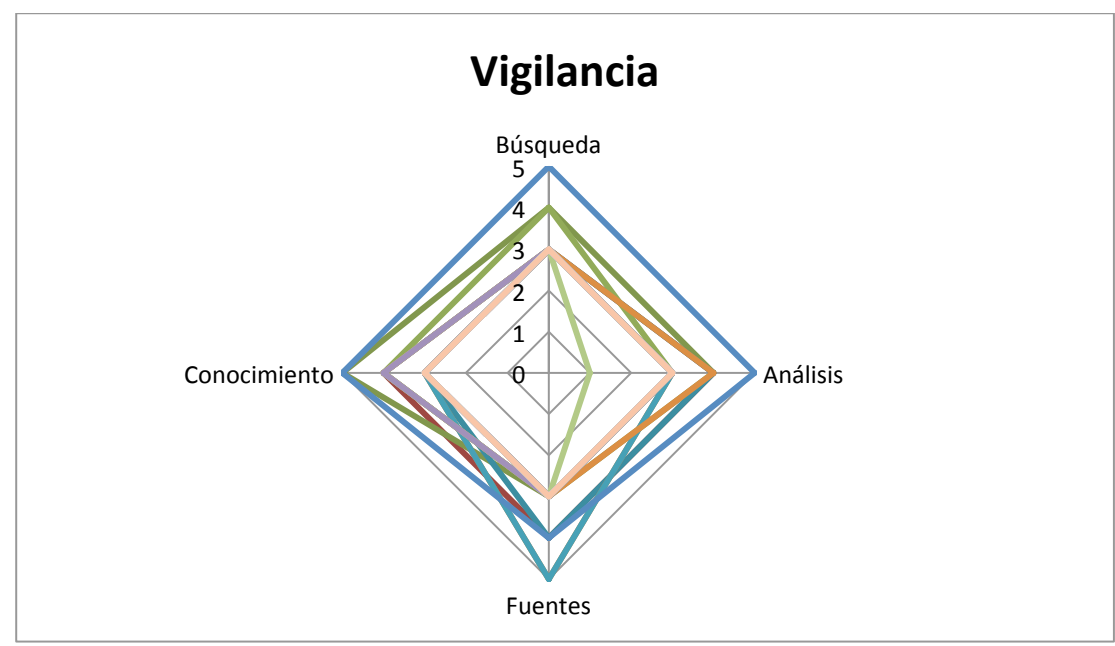

Fuente: Elaboración propia con base en los resultados del estudio

En la gráfica de Vigilancia Tecnológica (Figura 5), se denota el estrecho acercamiento que tiene la mayoría de las organizaciones hacia el centro de la gráfica, difícil es la situación que se presenta en las organizaciones con respecto a este tema, debido a que en muchas oportunidades realizan esta práctica por fuera de la ciudad o sencillamente existen casos de empresas que no dominan el concepto. En muchas otras situaciones, las empresas apuntan hacia el conocimiento y las fuentes de información sobre la competencia y muestran el uso del Internet como una herramienta normal de trabajo, pero no como elemento imprescindible para el estudio del entorno y las tendencias del mercado. Es decir, difícilmente practican la vigilancia tecnológica como estrategia para ganar en competitividad. 


\subsubsection{Análisis integrado del factor Gestión}

El análisis al grupo de empresas estudiado permitió comprobar que, en términos de toma de decisiones, existe una gran influencia del origen de la empresa y el nivel de dependencia que ésta tiene frente a una casa matriz fuera de la ciudad de Cartagena; en las agencias de aduanas de origen local y con alto grado de independencia de las decisiones hay un mayor grado de respuesta ante los cambios del entorno, mientras que el direccionamiento estratégico a nivel general en las empresas dependientes de una organización jerárquica superior, se encuentra supeditado a la dirección general y sus labores tienen un mayor grado de injerencia operativa.

En términos de las actividades de Investigación y Desarrollo, la principal herramienta de utilización por parte de las agencias de aduanas es la aplicación de las TIC dentro de su proceso, principalmente por las necesidades de comunicación con los clientes y la necesidad manifiesta de conocerlos. Sin embargo, no existe un proceso formal enfocado en el diseño y desarrollo de nuevos productos, esta circunstancia se tipifica al comprobar que el sector tiene una alta homogeneidad de necesidades de los clientes cuyos procesos ya se encuentran definidos y demarcados por ley. De igual forma, las actividades de Vigilancia Tecnológica y análisis del entorno se encuentran delimitados por procesos de seguimiento a sus competidores sin mayor profundidad en el análisis de tendencias.

Dentro del sector de agencias de aduanas, cabe destacar que los resultados evidencian que la infraestructura representa a nivel global un elemento dominante dentro de la estrategia de desarrollo de potencial competitivo en las organizaciones. El análisis de los resultados muestra la dinámica que tienen las empresas hacia la búsqueda de posicionamiento en el mercado a través de la oferta de una infraestructura ágil y acorde a los requerimientos del mismo.

El análisis de los factores de Vigilancia y toma de decisiones evidenció, además, que en el sector de las agencias de aduanas la construcción de conocimiento a partir de los datos del negocio, lo mismo que el análisis y procesamiento de los mismos para el desarrollo organizacional no son aplicados, centran la actividad de las empresas en la ejecución de operaciones establecidas.

\subsubsection{Factor de Posicionamiento Estratégico}

\subsubsection{Evaluación financiera}

De acuerdo a Porter, la estrategia empresarial integra diferentes elementos, dentro de los cuales se encuentra el cliente como eje central. Pero, al mismo tiempo, en su proceso de convertirse en líder del mercado, las empresas deben integrar decisiones de inversión y, por consiguiente, el análisis de dichos elementos como una variable dominante en las organizaciones (Porter, 2008). 
La estrategia empresarial involucra no sólo los factores analizados con anterioridad -que en muchos casos incluyen dentro de su evaluación la existencia de recursos económicos para el desarrollo de las actividades- sino que también requiere un espacio exclusivo para el análisis y toma de decisiones relacionados con los elementos financieros de las organizaciones.

Para el caso de la gráfica de evaluación financiera (Figura 6) se nota el interés que muestran las empresas del sector sobre el tema, se evidencia una alta concentración al nivel de finanzas, como un factor que resalta los resultados de acción en las organizaciones.

Sin embargo, el análisis de las cuatro dimensiones que componen la evaluación financiera dentro de las empresas, permite descubrir que no todas las organizaciones apuntan hacia el desarrollo del tema. Lo anterior puede explicarse por dos razones, la primera, porque muchas de las empresas analizadas realizan su proceso de forma sencilla y no existe una política definida sobre evaluación financiera. Además, en muchos casos las decisiones financieras y las evaluaciones son efectuadas desde la oficina principal en el interior o exterior del país. En la evaluación financiera sucede algo similar al factor anterior, a pesar de ser un factor de apoyo estratégico y de planeación es llamativo el hecho de que las empresas no se encuentren fortalecidas en tema de la evaluación financiera de mediano plazo y que todo se realice a través de la informalidad del día a día.

Figura 6. Evaluación financiera

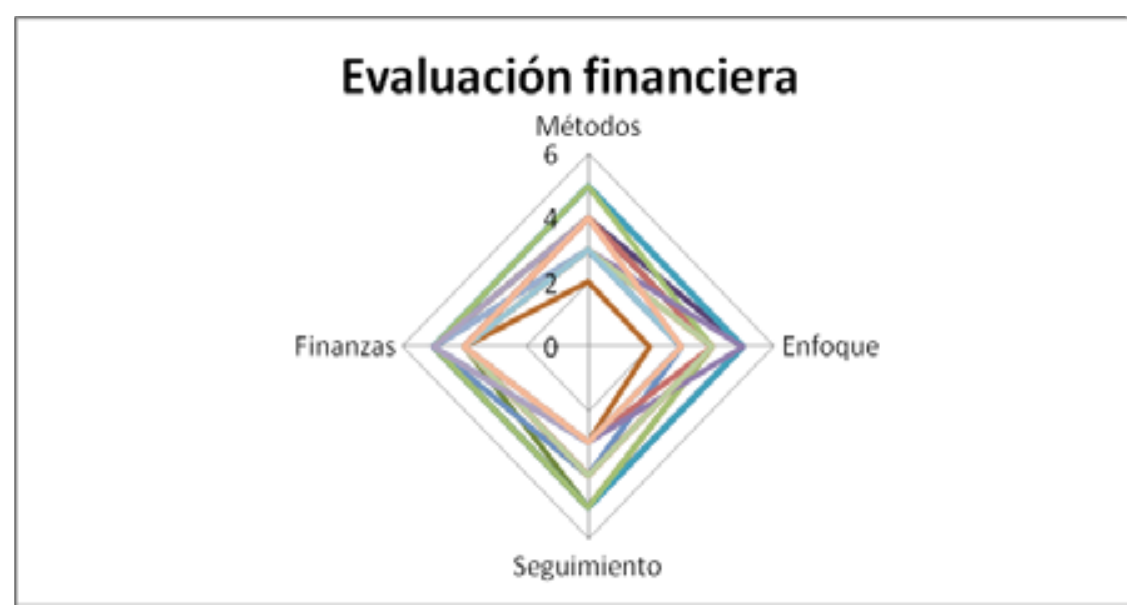

Fuente: Elaboración propia con base en los resultados del estudio 


\subsubsection{Posicionamiento}

Figura 7. Posicionamiento

\section{Posicionamiento}

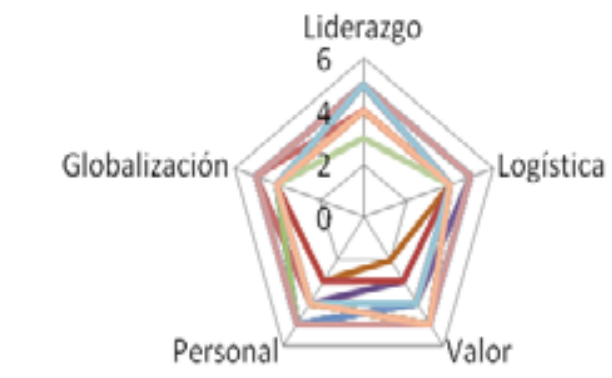

Fuente: Elaboración propia con base en los resultados del estudio

Para el caso de la gráfica de posicionamiento (Figura 7) se muestra cómo coinciden en gran manera las organizaciones en la búsqueda de este factor. Aunque no todas apuntan a la máxima calificación, si se muestra una intención significativa de todas en la búsqueda del liderazgo, la logística, la globalización y en garantizar que su personal sea idóneo y capaz de generar valor para la organización.

Este concepto está estrechamente relacionado con el buen nombre y la prestación del servicio, un factor clave de éxito al momento de competir en el mercado dentro del negocio de las agencias de aduanas.

En estas gráficas sobresalen las empresas de experiencia en el sector, con mayor número de personal y que, a su vez, se encuentran cualificadas para las gestiones encomendadas. Aunque se puede notar que se lucha por estar dentro del ranking, existen empresas que explotan de mejor forma su ventaja competitiva sobre las demás al mostrar su imagen ante propios y extranjeros. 


\subsubsection{Satisfacción del cliente}

En el análisis del comportamiento de esta variable dentro del proceso de estrategia competitiva, casi se nota un triángulo perfecto puesto que este tema es de los más mencionados a principios del siglo XXI. El concepto de satisfacción del cliente enmarca a todas las organizaciones, sin importar su situación en la economía. Es de suma importancia resaltar que las organizaciones ya son conscientes que los clientes son el alma de la organización y, por ello, se debe prestar el mejor de los servicios. En la gráfica se nota que, la gran mayoría de la muestra apunta hacia la consecución de los tres pilares con una leve variación que no es representativa de una tendencia.

En la Figura 8, se evidencia un alto grado de similitud porcentual con respecto al total de la muestra - con sesgos en su interior pero muy leves- lo cual permite afirmar que es, sin duda, una de las metas de las organizaciones de este siglo tener un buen SGC que permita generar valor intangible a sus clientes. En esta gráfica se muestra cómo las organizaciones tienen el concepto, pero no son del todo agresivas en el factor, se necesita mejorar.

Figura 8. Satisfacción del cliente

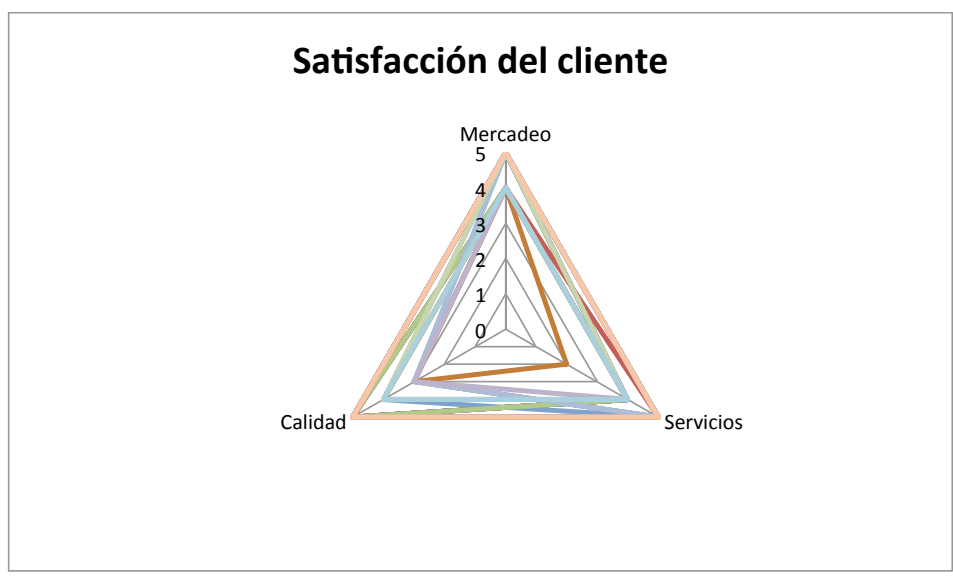

Fuente: Elaboración propia con base en los resultados del estudio

De igual forma cabe destacar, que al igual que el posicionamiento, esta variable tiene una relación directa con el cliente y los mecanismos de atención al mismo, que en un mercado con alto nivel de competencia, como son las agencias de aduanas, requiere estrategias directas que permitan mantener su fidelidad el mayor tiempo posible. 


\subsubsection{Planeación estratégica}

La planeación estratégica es clave en el proceso de direccionamiento de la organización hacia un futuro de interés para la misma. Estas acciones despliegan y requieren de una serie de recursos exclusivos para su labor (García, Bolivar, \& Roa, 2011).

Cabe destacar que no se persigue la existencia de un proceso formal de planeación, pero si se busca establecer hasta qué nivel dentro de las organizaciones existe el interés por dirigirse hacia un lugar común, dentro de un tiempo determinado.

Para este gráfico (Figura 9), la variación de las respuestas es notoria, entre 3 y 5 puntos con una empresa que manifestó no conducirse por ninguna visión en particular.

Figura 9. Planeación estratégica

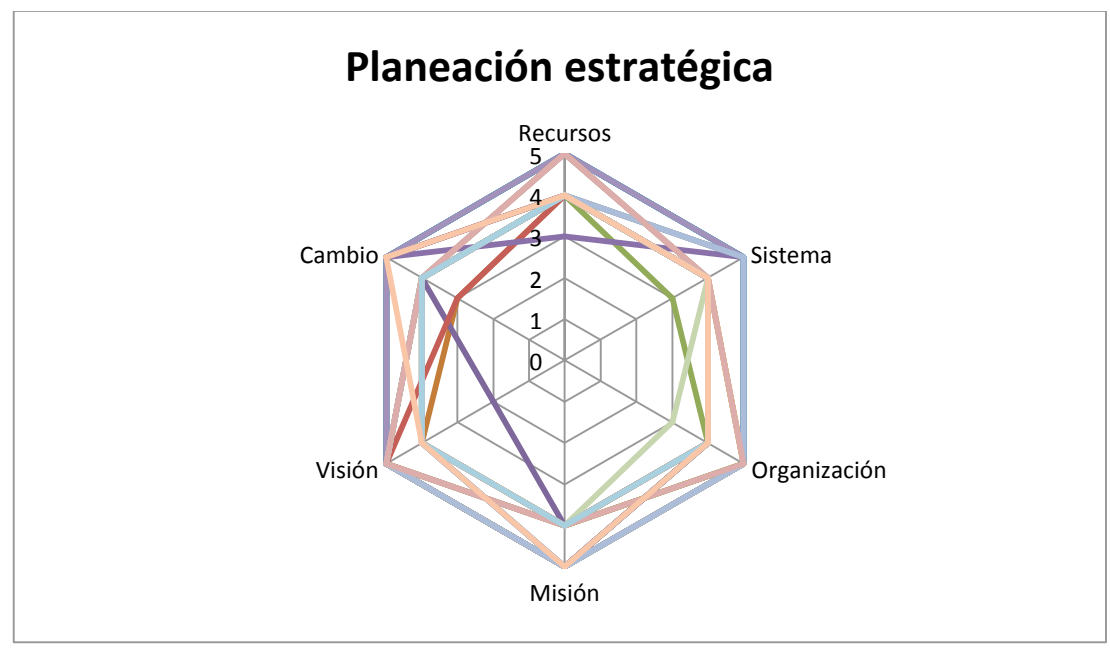

Fuente: Elaboración propia con base en los resultados del estudio

Otro elemento importante dentro de este factor para el análisis de las estrategias competitivas en las agencias de aduanas es la influencia de las organizaciones matriz, que se pone de manifiesto dentro de los mayores puntajes, mientras que hay una marcada estrategia de seguidor dentro de las organizaciones locales. 


\subsubsection{Análisis del factor de Posicionamiento Estratégico}

Para el caso de la evaluación financiera, se profundiza la evidencia que pone de manifiesto la ausencia de estrategias de construcción de conocimiento a partir de los datos, queda demostrado, en este caso específico, que en el nivel de políticas financieras, las agencias de aduanas adolecen de un direccionamiento enfocado más allá del estudio de sus resultados operativos.

A nivel de posicionamiento, por otra parte, al igual que en la variable de satisfacción del cliente, existe una alta tendencia de las empresas hacia darle un mayor nivel de importancia. El alto grado de competencia dentro del sector, puede ser uno de los desencadenantes de la concentración de estrategias en este nivel. Por su parte, en el análisis de los procesos de planeación estratégica los resultados muestran que, al igual que en el factor de gestión, hay una fuerte influencia de esta variable sobre las empresas con un liderazgo no local, mientras que las otras concentran sus tareas en el desarrollo de funciones operativas.

\subsubsection{Análisis Cuantitativo y Evaluación Conjunta de Factores}

Tal como se planteó al inicio del artículo, cuando se describió el proceso metodológico que orienta este estudio, la evaluación individual de los factores con el método gráfico persigue, a nivel general, establecer los parámetros cualitativos que orientan las acciones de estrategia competitiva de las organizaciones. En esta sección, a partir de la calificación reducida, se persigue establecer una visión general del modelo y la confluencia de esfuerzos de las empresas en torno a una estrategia particular.

Para el análisis cuantitativo se definió como eje central del proceso el área encerrada por el perímetro de las evaluaciones en cada figura radial. El cálculo del área encerrada en el perímetro de la figura radial, da como resultado un valor numérico que representa la calificación obtenida por el componente, dicha calificación ha sido convertida en dos tipos de medida, la calificación completa (Acom), que es el valor numérico obtenido directamente en la gráfica y la calificación reducida (Ared), que representa el porcentaje del área de gráfico que ha alcanzado dicha calificación respecto al puntaje máximo posible. La calificación completa, permite obtener el valor global del área encerrada por la conjunción de los diferentes valores de cada componente, mientras que la calificación reducida - que es la que interesa a las empresas - les permite establecer la distancia que existe entre su realidad y el estado ideal de cada componente

Para obtener la calificación completa de cada componente se aplica la siguiente fórmula: 


$$
A \operatorname{com}=\frac{\operatorname{Sin} \theta}{2}\left(\left(\sum_{i=1}^{j-1}\left(V_{i} * V_{i-1}\right)+\left(V_{j} * V_{i}\right)\right)\right)
$$

(1)

Donde

$\mathrm{J}=\mathrm{El}$ número de variables de cada componente

$\mathrm{V}=\mathrm{El}$ valor obtenido en la calificación que cada componente i

$\theta=\frac{360}{j}$ Convertido a Radianes

(2)

La calificación reducida es:

Ared $=\frac{\text { Acom }}{\text { Aopt }}$

(3)

Donde

Aopt $=$ Es el valor óptimo de cada componente en la evaluación

Para lograr este propósito se ha desarrollado una gráfica general que muestra para cada una de las 30 empresas evaluadas los resultados porcentuales de calificación a nivel de los factores, se busca identificar de manera visual la existencia de elementos estratégicos comunes.

A nivel general, el análisis gráfico permite identificar que el desarrollo de las estrategias no sigue un hilo conductor común (Figura 10), que evidencia la presencia de diferentes planteamientos y posiciones dentro del sector. En el campo de vigilancia, por ejemplo, es posible observar que hay una confluencia hacia la obtención de bajas calificaciones por debajo de un rendimiento del $50 \%$ en la estrategia. Sin embargo, resaltan algunas empresas con valores superiores a la media. 
Figura 10. Modelo general por factores.

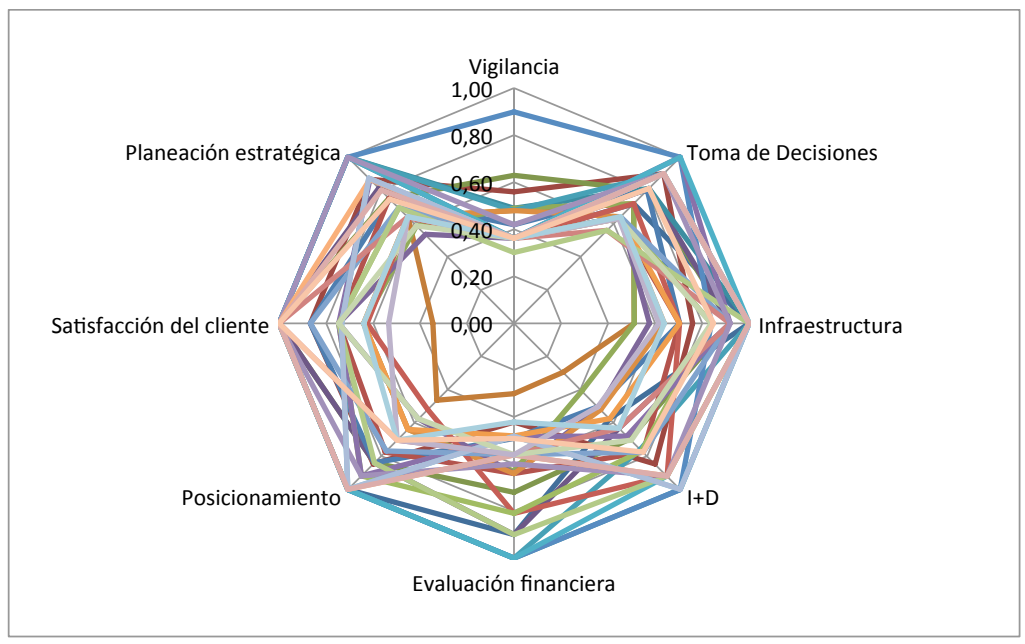

Fuente: Elaboración propia con base en los resultados del estudio

En los casos de planeación estratégica, toma de decisiones, infraestructura, posicionamiento e $\mathrm{I}+\mathrm{D}$, se observa una alta concentración de estrategias de acción con evaluaciones superiores al 40\%, y en los casos de satisfacción del cliente y evaluación financiera se observa, a su vez, una alta dispersión.

El resultado de este análisis no es concluyente con la hipótesis de concurrencia de las estrategias planteada en la sección metodológica. Sin embargo, debido a la alta superposición evidente dentro del estudio, que no muestra a primera vista la existencia de una estrategia dominante, los resultados obtenidos se sometieron a un proceso de evaluación estadística basado en análisis de correspondencias, para comprobar (o rechazar) las hipótesis relacionadas con las estrategias encontradas.

La Figura 11 - elaborada a partir del análisis de correspondencias de la evaluación de los componentes de estrategia - muestra un alto grado de variabilidad y dispersión en la concurrencia de sus estrategias, lo que implica que las empresas siguen ritmos individuales, aunque tienen una tendencia a seguir estrategias similares a algunos de sus competidores, lo que podría denominarse como estrategias referenciadas. 
Figura 11. Gráfico de correlación de estrategias por empresas

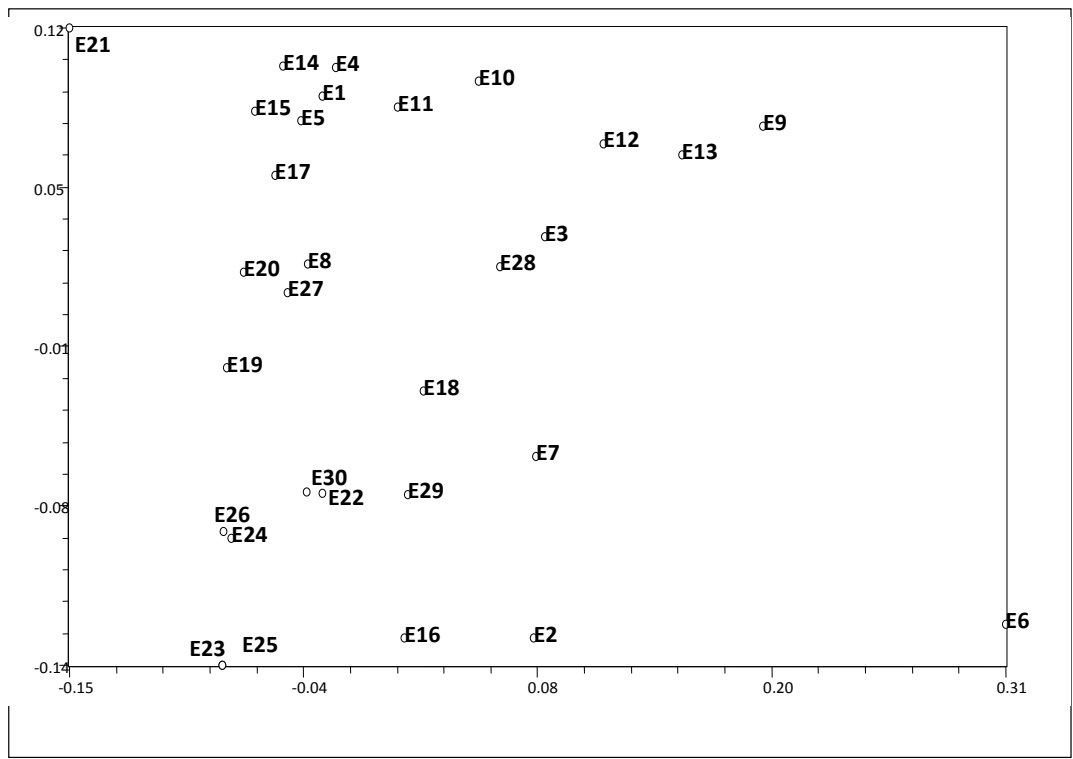

Fuente: Elaboración propia con base en los resultados del estudio

A partir de las actividades desarrolladas y los resultados obtenidos, en este apartado es posible apreciar que la dispersión en el desarrollo estratégico se fundamenta principalmente en las diferencias marcadas en el sistema orgánico y de dirección de su estructura gerencial.

De igual forma, el análisis se condujo hacia la evaluación de concurrencia de los factores. La Figura 12 muestra un mapa de correspondencias aplicado a las evaluaciones de los factores que definen los elementos de la estrategia de potencial competitivo. 
Figura 12. Gráfico de correlación de estrategias por variables

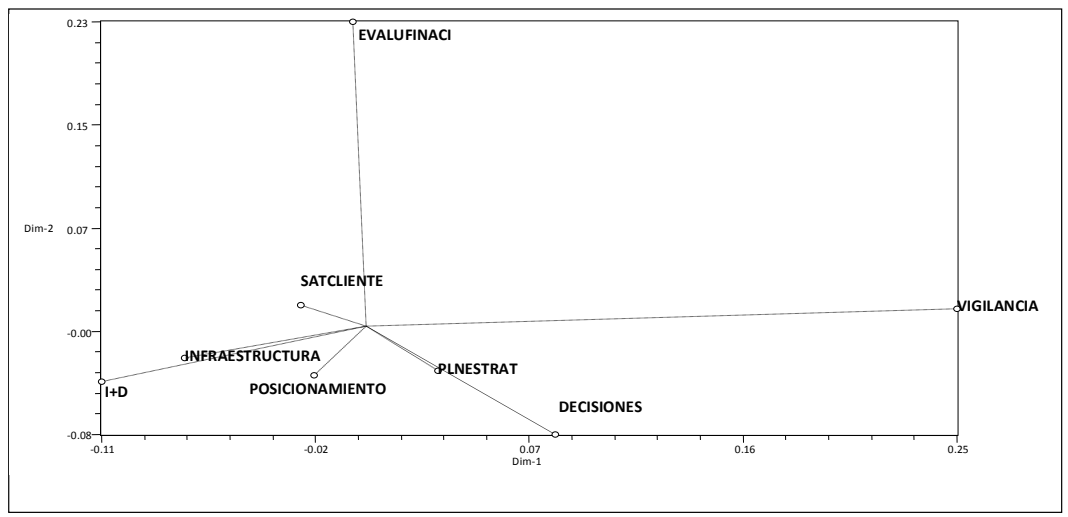

Fuente: Elaboración propia con base en los resultados del estudio

En esta gráfica se analiza, en términos generales, de qué manera se agrupan los datos, en torno a estrategias definidas. Para el caso de este análisis de correspondencia se parte del factor competitividad- que en este caso corresponde a la variable dependiente del estudio - y a su alrededor se mueven sub-factores que determinan su estabilidad, es decir, su crecimiento o anulación del sistema. Se nota de manera clara cómo alrededor de la competitividad convergen los factores estudiados y se muestran exactamente los mismos resultados que los individuales del análisis cualitativo. Aquí se observan tres elementos que interactúan de forma importante para las organizaciones y sobre los que todos trabajan o trabajaron para ser más competitivas. Estos tres criterios son: Infraestructura, Posicionamiento y Satisfacción del cliente.

De igual forma, las variables Planeación Estratégica y Toma de Decisiones se ubican cercanos al conglomerado principal. Se han definido, para efecto de las conclusiones, estos dos elementos como de soporte, ya que brindan apoyo a la gestión de las organizaciones dentro de su estrategia competitiva, aunque son generadores de variación en la misma debido principalmente, y como ya se ha comentado, a las diferentes denominaciones estructurales presentes dentro del entorno de las agencias.

También se muestran tres elementos dispersos que representan los factores en los que se necesita trabajar para mejorar la medición de la competitividad en el sector, estos picos alejados son: Vigilancia Tecnológica, I+D y Evaluación Financiera. Tal como se pudo apreciar en la evaluación cualitativa, estos tres elementos necesitan de especial cuidado y requieren la realización de esfuerzos importantes con el fin de lograr el mejoramiento de estas arterias neuronales del sistema logístico de la ciudad de Cartagena. 
Alomoto Bansui, N. R. (2010). Competitividad, Pymes y Stakeholders. XLV Asamblea CLADEA 2010 (pág. 10). Cartagena de Indias: CLADEA.

Esser, K., Hillebrand, W., Messner, D., \& Meyer-Stamer, J. (1996). Competitividad sistémica: Nuevo desafío a las empresas y a la política. Revista de la CEPAL, $39-52$.

García, G., Bolívar, J., \& Roa, R. I. (2011). Gerencia Integral para el siglo XXI. Prácticas para mejorar la competitividad de las organizaciones. Bogotá D.C: Universidad EAN.

Krugman P. (1994), "Strategic trade policy and the new international economics", The MIT Press

Michalet, C. (1981): “Competitiveness and internationalization”, (mimeo), OECD, París

Nurbel, Alain (2007) The global competitiveness of the nation: a conceptual discussion. Journal of business and economics research. Vol. 5 No. 10

Porter, M. (2008). Ser competitivo. Barcelona: Planeta.

Raymond, L., \& St-Pierre, J. (2010). R \& D as a determinant of innovation in manufacturing SMEs: An attempt at empirical clarification. Technovation, 30 (1), 48-56.

Ríos Montañez, R. O. (2011). Metodología de análisis financiero para PYMES. Universidad de las Américas, Programa de Administración de empresas. Puebla: Escuela de negocios y economía.

Sarmiento Paredes, S., Sánchez Sánchez, A., \& Cruz García, M. G. (2009). Competitividad y desarrollo sustentable empresarial. Revista Internacional, la nueva gestión organizacional, 112-134.

Sepúlveda Chaverra, J. D., Meriño Stand, L. I., \& Angulo Cuentas, G. L. (2010). Vigilancia tecnológica para el desarrollo empresarial. Cartagena de Indias: Fundación Universitaria Tecnológico Comfenalco.

Sepúlveda Chaverra, J. D., Meriño Stand, L. I., Del Río Cortina, J. L., \& García, A. (2010). Metodología para la evaluación del potencial competitivo en pequeñas y medianas empresas basado en el sistema de capacidades internas. Revista internacional Administración y Finanzas, 97-107. 